\title{
Les relations entre la grande distribution et ses fournisseurs : bilan et limites de trente ans de régulation
}

\author{
Marie-Laure ALLAIN ${ }^{1}$ et Claire CHAMBOLLE ${ }^{2}$
}

\section{Introduction}

Depuis l'ouverture du premier hypermarché en 1963, la grande distribution se trouve régulièrement placée au cœur de débats qui retiennent l'attention des médias, de l'opinion publique, et des gouvernements. Les récentes opérations de concentration ont modifié les structures du marché de la distribution. En l'an 2000, la fusion des groupes français Carrefour et Promodès a donné naissance au second groupe mondial du secteur, et à l'une des plus grandes entreprises françaises, dont le chiffre d'affaires annuel dépasse 50 milliards d'euros. Bien implantés à l'étranger, et développant rapidement leur stratégie d'internationalisation, les grands distributeurs français sont devenus des acteurs majeurs de l'économie nationale. Cependant, ce développement du secteur de la grande distribution engendre des conflits fréquents à la fois avec les fournisseurs et les formes concurrentes de commerce. Ainsi sont portés à l'arbitrage des pouvoirs publics les concentrations de centrales d'achat ou de groupes de distribution, mais aussi les conflits entre fournisseurs et grands distributeurs. Les Assises de la Distribution, qui se sont tenues le 13 janvier 2000 à Paris, avaient pour objectif de susciter un débat sur les relations entre la grande distribution et ses fournisseurs, dont le déséquilibre avait été mis en évidence dans le rapport parlementaire ${ }^{3}$ qui préparait cette manifestation.

En effet, le développement de la grande distribution a profondément modifié les relations entre industrie et commerce ${ }^{4}$, et a contribué à déplacer le pouvoir de marché vers les distributeurs. En France, les producteurs disposant d'une marque connue des consommateurs ont été pendant longtemps en position de force vis-à-vis d'un secteur commercial constitué de petites entreprises. Ce rapport de forces s'est généralement conservé pour les catégories de produits concernées par la distribution sélective ${ }^{*^{5}}$ ou exclusive*, mais, pour les produits de consommation courante*, l'absence de circuit de distribution de ce type impose aux producteurs de commercialiser leur production par le biais d'une grande distribution de plus en plus puissante, et dont les enseignes deviennent de véritables marques.

\footnotetext{
${ }^{1}$ CNRS, Laboratoire d'Econométrie de l'Ecole Polytechnique et CREST-LEI. Adresse : 1 rue Descartes, 75005 Paris, tel : 0155558541 , marie-laure.allain@polytechnique.fr.

${ }^{2}$ INRA-LORIA et Laboratoire d'Econométrie de l'Ecole Polytechnique. Adresse : 65 Boulevard de Brandebourg, 94205 Ivry sur Seine, tel : 01-49-59-69-46, chamboll@,ivry.inra.fr.

| ${ }^{3}$ Le rapport dit « Charié-Le Déaut_, N²072, publié en février 2000, version préliminaire de janvier 2000.

${ }^{4}$ Pour une analyse des rapports de force entre producteurs et distributeurs, voir Allain (2000) et Chambolle (2000).

${ }^{5}$ Les mots suivis d'une astérisque sont définis dans le glossaire à la fin du texte.
} 
De fait, la puissance d'achat des grands distributeurs, c'est-à-dire leur pouvoir de marché en tant qu'acheteurs, augmente constamment ${ }^{6}$. La concentration croissante du secteur de la distribution par le biais des fusions et acquisitions est encore renforcée par la constitution de centrales d'achat : des distributeurs se regroupent pour s'approvisionner collectivement, afin d'accroître leur pouvoir de négociation avec leurs fournisseurs, en constituant ces centrales dont le rôle est de négocier les conditions commerciales avec les producteurs. Actuellement, cinq centrales d'achat réalisent presque $95 \%$ du chiffre d'affaires de la grande distribution : dans ce cadre typiquement oligopolistique, les problèmes de concurrence prennent une importance particulière, et appellent la vigilance des pouvoirs publics.

D'autre part, le développement ${ }^{7}$ des marques de distributeurs* (marques propres à une enseigne) a également contribué à la modification des rapports de force entre producteurs et distributeurs. Le pouvoir de négociation des détaillants s'est accru, et ils sont parfois allés jusqu'à prendre le contrôle de producteurs auxquels ils peuvent sous-traiter la fabrication de produits qu'ils conçoivent eux-mêmes. Ainsi, l'apparition des marques de distributeurs (qui représentaient 20,3\% du chiffre d'affaires des hypermarchés et supermarchés en 1998, d'après Eurostaf) contribue à créer des structures verticales complexes, dans lesquelles un distributeur peut intervenir en amont du producteur, par exemple en concevant le produit et en imposant les méthodes de fabrication : ainsi par exemple, « la frontière entre production et distribution est devenue floue» dans les filières de l'habillement (Philippe, 1997). L'importance croissante des primes de référencement* dans les budgets commerciaux, et plus généralement l'accroissement des marges arrière*, constitue un autre exemple de cette modification $^{8}$. Ce renversement apparent des rapports de force présente un intérêt tant sur le plan économique que juridique, et incite à s'interroger sur l'efficacité des outils élaborés par les pouvoirs publics pour contrôler les déséquilibres qu'une telle situation peut engendrer.

Nous proposons dans cet article d'examiner la façon dont la réglementation s'est adaptée à l'évolution du secteur de la distribution et à l'émergence de nouvelles problématiques, notamment en ce qui concerne les rapports de force entre les acteurs du marché. En France, les relations contractuelles entre producteurs et distributeurs relèvent à la fois du droit commercial et du droit de la concurrence, ce qui contribue à créer un environnement juridique parfois complexe. Nous présentons le cadre légal et réglementaire en distinguant les « règles de comportement», des «règles de structure ». Les règles de comportement définissent le cadre des pratiques autorisées dans la concurrence entre distributeurs, ou dans les négociations entre producteurs et distributeurs. Elles sont principalement énoncées dans le code de commerce, dont la partie législative synthétise, dans son livre IV intitulé « de la liberté des prix et de la concurrence », les dispositions de l'ordonnance de 1986, qui posait les

\footnotetext{
${ }^{6}$ Pour une étude de la puissance d'achat des distributeurs en Europe, voir Dobson et al. (1999).

${ }^{7}$ Pour une analyse théorique du développement des marques de distributeurs, voir Mills (1995), Bontems, Monnier et Réquillart (1999), Berges-Sennou (2001) ou Caprice (2000).

${ }^{8}$ Bien que leur montant soit difficile à connaître exactement, l'ILEC estime que les marges arrière représentaient en 1999 pour les produits

d'épicerie 88\% de la marge totale des distributeurs, contre 74\% en 1995.
} 
fondements du droit de la concurrence français, de la loi Galland de 1996, et d'une partie de la loi sur les nouvelles régulations économiques ${ }^{9}$ de 2001 . Par règle de structure, nous entendons pour l'essentiel, les mesures qui permettent aux pouvoirs publics de modifier ou de contrôler l'évolution du réseau de distribution. Elles regroupent des mesures aussi diverses que le dispositif de contrôle des concentrations, ou la loi Raffarin de 1996. Nous évoquerons à la fois la réglementation portant spécifiquement sur les relations verticales, et certaines règles portant sur les relations horizontales, dans la mesure où elles peuvent influer indirectement sur les relations entre producteurs et distributeurs. En explicitant les effets directs ou indirects des diverses mesures adoptées sur les relations entre industrie et commerce, nous donnons des éléments d'appréciation de l'écart existant entre les objectifs affichés et les résultats atteints par le dispositif réglementaire et légal.

Nous présentons dans cet article le droit français, bien que les entreprises françaises soient également soumises à la loi communautaire. En effet, les pratiques anticoncurrentielles et les comportements abusifs des entreprises françaises peuvent être soumis à la fois aux règles du droit de la concurrence français, et aux règles européennes exprimées dans le Traité de Maastricht entré en vigueur le ler novembre 1993. Le champ d'application des règles communautaires ou nationales n'est pas toujours très clairement délimité, mais une règle de primauté du droit communautaire existe. D'après les articles 81 et 82 du Traité de Maastricht $^{10}$, la Commission est compétente pour juger les faits susceptibles d'affecter le commerce entre Etats membres, alors que la compétence des autorités nationales se limite à ce qui relève des effets sur l'économie nationale. Les deux juridictions peuvent en fait intervenir parallèlement : lorsqu'une décision communautaire a été rendue sur certains faits, les autorités nationales restent compétentes pour appliquer le droit national aux mêmes faits ${ }^{11}$, dans la mesure où l'application du droit national « ne peut porter préjudice à l'application pleine et uniforme du droit communautaire, et à l'effet des actes d'exécution de celui-ci ». Cependant le droit français de la concurrence s'est assez largement inspiré du droit européen. Nous signalerons au cours de la présentation les différences majeures entre les règles nationales et communautaires.

\section{Les règles de comportement}

Le comportement des producteurs et des distributeurs est soumis à un ensemble de règles visant à garantir le respect de la concurrence. Nous présentons dans un premier temps les règles générales du droit de la concurrence, puis nous reviendrons sur les modifications apportées en 1996 par la loi Galland dans l'optique de la régulation des relations entre industrie et commerce.

\footnotetext{
${ }^{9}$ Loi n²001-420 du 15 mai 2001 sur les nouvelles régulations économiques. Nous choisissons, dans cet article, une présentation chronologique des textes de lois, afin de suivre l'évolution des réformes, mais nous présentons systématiquement la correspondance entre les références des lois et la nouvelle nomenclature du code de commerce.

${ }^{10}$ Ces articles reprennent les articles 85 et 86 du Traité de Rome. Pour une présentation du débat sur le champ d'application des règles communautaires, voir Boutard-Labarde et Canivet (1994).
} 


\section{Le cadre général}

L'ordonnance n86-1243 du 1er décembre 1986, que nous appellerons simplement dans la suite du texte «ordonnance de 1986 », pose les jalons du droit de la concurrence français actuel. Elle pose la liberté des prix et de la concurrence comme principe général, crée le Conseil de la Concurrence, dont le rôle est de juger et condamner les pratiques anticoncurrentielles pouvant affecter l'économie, et établit les règles de contrôle des concentrations. La mise en œuvre du droit de la concurrence relève à la fois du Conseil de la Concurrence et des juridictions de droit commun. Le Conseil de la Concurrence ne peut être saisi que de faits constitutifs de pratiques anticoncurrentielles, et n'est pas compétent pour statuer sur les pratiques restrictives, qui sont $\mathrm{du}$ ressort des juridictions civiles ou commerciales. Cependant, les tribunaux civils ou commerciaux peuvent soumettre ces pratiques à son appréciation, si elles émanent manifestement d'une entente, d'un abus de position dominante ou d'un abus de dépendance économique. En outre, le Conseil peut être consulté pour avis par le gouvernement, les juridictions ou diverses institutions, sur toutes les questions de concurrence.

\section{Les accords anticoncurrentiels et les restrictions verticales}

Les pratiques anticoncurrentielles et restrictives condamnables sont définies respectivement dans les titres II et IV du livre IV du code de commerce partie législative, qui reprend essentiellement les dispositions de l'ordonnance de 1986 (titres III et IV). Ces règles générales s'appliquent au cas particulier des relations entre producteurs et distributeurs. De nombreuses restrictions verticales peuvent être condamnées au titre de l'article L420-1 du code de commerce, qui traite des ententes et des pratiques anticoncurrentielles concertées :

«Sont prohibées [...], lorsqu'elles ont pour objet ou peuvent avoir pour effet d'empêcher, de restreindre ou de fausser le jeu de la concurrence sur un marché, les actions concertées, conventions, ententes expresses ou tacites ou coalitions, notamment lorsqu'elles tendent à :

1. Limiter l'accès au marché ou le libre exercice de la concurrence par d'autres entreprises ;

2. Faire obstacle à la fixation des prix par le libre jeu du marché en favorisant artificiellement leur hausse ou leur baisse ;

3. Limiter ou contrôler la production, les débouchés, les investissements ou le progrès technique ;

4. Répartir les marchés ou les sources d'approvisionnement ».

Ainsi, les pratiques de prix de revente imposé ou les restrictions quantitatives (quotas) sont directement visées par cet article. L'article suivant (L420-2) définit la notion de pratiques abusives $^{12}$ :

«Est prohibée [...] l'exploitation abusive par une entreprise ou un groupe d'entreprises d'une position dominante sur le marché intérieur ou une partie substantielle de celui-ci. Ces abus

\footnotetext{
${ }^{11}$ Par exemple, une entente entre entreprises françaises dans le secteur des lasers pulsés a été condamnée à la fois par la Commission européenne (Décision du 27/07/1992) et par le Conseil de la Concurrence (Décision n93-D-11 du 4 mai 1993).

${ }^{12}$ Ces dispositions sont complétées par l'article 1382 du code civil, qui définit les actes de "concurrence déloyale », en particulier les détournements de clientèle par «manoeuvre, tromperie, dénigrement ou publicité fallacieuse ».
} 
peuvent notamment consister en refus de vente, en ventes liées* ou en conditions de vente discriminatoires ainsi que dans la rupture de relations commerciales établies, au seul motif que le partenaire refuse de se soumettre à des conditions commerciales injustifiées.

Est en outre prohibée, dès lors qu'elle est susceptible d'affecter les fonctionnement ou la structure de la concurrence, l'exploitation abusive par une entreprise ou un groupe d'entreprises de l'état de dépendance économique dans lequel se trouve à son égard une entreprise cliente ou fournisseur. Ces abus peuvent notamment consister en refus de vente, en ventes liées ou pratiques discriminatoires [...]. »

Cependant, les pratiques résultant de l'application d'un texte législatif spécifique, ou les pratiques «dont les auteurs peuvent justifier qu'elles ont pour effet d'assurer un progrès économique », ne sont pas soumises à ces dispositions (article L420-4). Par ailleurs, des exemptions par catégorie peuvent être accordées par décret et appliquées collectivement à certaines catégories d'accords : rares en France, mais plus développées dans le droit européen, elles constituent une des principales différences entre les droits français et communautaire. La Commission européenne a récemment adopté des mesures visant à harmoniser les règles d'exemption applicables aux restrictions verticales ${ }^{13}$. Ce nouveau texte remplace notamment les règlements d'exemption concernant la distribution sélective ou les contrats de franchise. Il fait intervenir des seuils de parts de marché en deçà desquels la plupart des restrictions verticales sont autorisées (à l'exception de certaines restrictions fondamentales comme les prix imposés ou l'exclusivité territoriale). Au-delà de ces seuils, les restrictions ne sont pas interdites per se mais font l'objet d'un contrôle au cas par cas.

Par ailleurs, les contrats d'exclusivité et l'octroi de territoires exclusifs ne sont pas interdits per se et sont traités au cas par cas, mais ils sont souvent considérés comme des ententes anticoncurrentielles et condamnés au titre de l'article L420-1. Enfin, les pratiques discriminatoires, qui recouvrent des comportements très divers, sont condamnées, qu'elles proviennent de producteurs ou de commerçants : il est interdit « de pratiquer, à l'égard d'un partenaire économique, ou d'obtenir de lui des prix, [...] des conditions de vente [...] ou d'achat discriminatoires et non justifiés par des contreparties réelles en créant, de ce fait, pour ce partenaire, un désavantage ou un avantage dans la concurrence ». Notons que les condamnations les plus fréquentes dans le secteur de la distribution concernent des pratiques discriminatoires.

La législation française interdit certaines restrictions verticales per se (titre IV du livre IV du code de commerce) : pour les condamner, les instances compétentes n'ont pas à prouver qu'elles ont « eu pour objet ou pour effet d'empêcher, de restreindre ou de fausser le jeu de la concurrence ». Ainsi, la fixation par les producteurs de prix planchers (et a fortiori celle des prix de revente imposés), les ventes liées ou les quotas sont prohibés. Les centrales d'achat ne

\footnotetext{
${ }^{13}$ Le règlement $\mathrm{CE} \mathrm{n}{ }^{\circ} 2790 / 1999$ du 22 décembre 1999 constitue un ensemble de règles générales applicable à tous les accords verticaux. En outre, le règlement d'exemption accordé jusqu'alors à la distribution automobile doit être modifié en 2002.
} 
sont pas non plus autorisées à fixer les prix de revente de leurs adhérents ${ }^{14}$. La revente à perte est également interdite aux commerçants.

\section{L'abus de position dominante}

Le fait, pour une entreprise ou un groupe d'entreprises, de disposer d'une position dominante sur un marché, n'est pas condamnable ${ }^{15}$ en soi : seul l'abus d'une position dominante est prohibé par l'article L420-2 du code de commerce. Cette notion d'abus est assez floue, et requiert un traitement au cas par cas. Ainsi, certaines pratiques qui ne seraient pas considérées comme anticoncurrentielles si elles émanaient d'entreprises soumises à une forte concurrence, sont considérées comme telles si elles émanent d'entreprises en "position dominante ». Le texte ne précise cependant pas ce qui est entendu par ce terme. La définition généralement retenue est celle énoncée par la Cour de Justice Européenne dans l'arrêt relatif à l'affaire United Brands du 14/02/1978: «la position dominante [...] concerne une situation de puissance économique détenue par une entreprise qui lui donne le pouvoir de faire obstacle au maintien d'une concurrence effective sur le marché en cause et lui fournit la possibilité de comportements indépendants dans une mesure appréciable vis-à-vis de ses concurrents, de ses clients, et, finalement, des consommateurs ». Ainsi, la notion juridique de position dominante rejoint la notion économique de pouvoir de marché, une entreprise en position dominante n'étant pas affectée, dans son comportement et ses choix stratégiques, par la concurrence des autres entreprises, et pouvant au contraire influencer le comportement de ses concurrents.

Pour qu'une entreprise soit sanctionnée au titre de l'article L420-2, le Conseil de la Concurrence doit avant tout définir le marché pertinent ${ }^{16}$ sur lequel l'entreprise est active, puis démontrer sa position dominante, et enfin prouver le caractère abusif et anticoncurrentiel de ses pratiques. En ce qui concerne les producteurs, le marché pertinent est le plus souvent défini selon des critères qualitatifs ou quantitatifs de substituabilité des produits. En revanche, la délimitation du marché pertinent dans le cadre de la distribution est plus délicate, car le caractère multi-produits de l'activité engendre de nombreuses possibilités d'interprétations. En général, la définition retenue repose essentiellement sur des critères géographiques, et le marché pertinent est défini en délimitant les zones de chalandise* en fonction du temps de déplacement des clients. D'autres facteurs peuvent intervenir de façon complémentaire dans la définition du marché pertinent, par exemple le format de distribution, les gammes de produits offertes, la présence de marques de distributeurs, ou la nature des services offerts par les points de vente considérés, mais il est plus difficile de les prendre en compte. Une fois le marché pertinent délimité, la part de marché est bien entendu l'indicateur de position dominante le plus couramment utilisé. Ainsi, une entreprise dont la part de marché dépasse $50 \%$ peut être présumée disposer d'une position dominante. Cependant, une part de marché

\footnotetext{
${ }^{14}$ Voir par exemple la décision 97-D-49 du Conseil de la Concurrence, relative a des pratiques mises en œuvre sur le marche de l'optique médicale.

${ }^{15}$ On verra toutefois dans la suite du texte que, dans le cadre du contrôle des concentrations, la constitution d'une position dominante par croissance externe peut susciter l'interdiction de l'opération de concentration.

${ }^{16}$ Pour une discussion sur les méthodes de délimitation du marché pertinent, voir Philippe (1998).
} 
élevée est un indice nécessaire mais non suffisant de position dominante; en outre, aucun seuil de part de marché précis n'est défini ${ }^{17}$. D'autres facteurs entrent en jeu, comme la structure du marché, son degré de concentration, l'existence de barrières à l'entrée, l'appartenance de la firme à un groupe puissant, ou encore la notoriété du produit, de la marque ou de l'enseigne. Ainsi, une entreprise disposant d'une part de marché de $30 \%$ n'est pas dans la même situation si deux entreprises se partagent les $70 \%$ de parts de marché restantes, ou si le reste du secteur est atomistique. La situation concurrentielle est, là encore, déterminante, et doit être appréciée par les autorités afin de juger si l'entreprise est en situation dominante. Pour établir l'existence d'une position dominante, les autorités prennent généralement en compte des critères structurels (comme les parts de marché) et comportementaux (par exemple l'adoption de stratégies de prédation...).

Enfin, lorsque la position dominante est établie, les autorités doivent juger si le comportement de l'entreprise relève d'un abus. Les comportements abusifs peuvent recouvrir de nombreuses pratiques, visant soit à nuire directement aux concurrents, ou à empêcher l'entrée de nouveaux concurrents, soit à obtenir des clients ou fournisseurs des conditions qu'une situation de concurrence effective n'aurait pas permis d'obtenir. Certaines pratiques tarifaires qui tendent à modifier artificiellement les prix sont couramment considérées comme abusives. Ainsi, la pratique d'un prix excessif par une entreprise en situation de position dominante, par exemple en monopole, peut revêtir un caractère abusif. A l'inverse, la pratique d'un prix prédateur ${ }^{18}$ peut également être condamnée à ce titre, car elle vise à long terme la disparition de la concurrence. En outre, les conditions de vente discriminatoires sont prohibées, lorsqu'elles ne sont pas justifiées par des différences de coûts. De plus, certaines pratiques révélant un abus de position dominante dans les relations commerciales sont explicitement interdites. Ainsi, le refus de vente ou de prestations de service était interdit per se par la législation française jusqu'à ce que la loi Galland de 1996 supprime cette interdiction. En outre, la « rupture des relations commerciales avec un partenaire économique sans motif valable » est interdite, et concerne aussi bien la cessation des ventes de la part d'un fournisseur, que la rupture de référencement de la part d'un distributeur. Cette interdiction devrait a priori suffire à empêcher les distributeurs d'utiliser les menaces de déréférencement comme arme dans les négociations avec leurs fournisseurs, mais l'ordonnance de 1986 ne permettait de condamner une telle rupture des relations commerciales que si elle était le fait d'une entreprise dont on peut prouver qu'elle occupe une position dominante $^{19}$ : la loi Galland a apporté des modifications sur ce point.

\footnotetext{
${ }^{17}$ Dans certains cas, comme dans la Décision n ${ }^{\circ} 91-D-12$ du 26/03/1991, le Conseil de la Concurrence a jugé qu'une part de marché de plus de $50 \%$ n'était pas associée à une position dominante. Cependant, de manière générale, des parts de marché supérieures à $70 \%$ ou $80 \%$ sont automatiquement considérées comme des indices de position dominante.

${ }^{18}$ Pour une analyse économique des stratégies de prédation, voir Rey, P. et J. Tirole (1997).

${ }^{19}$ Ainsi, la société Primistères Reynoird a été condamnée en 1997 au titre de l'article 8.1 de l'ordonnance de 1986 (article L420-2 du code de commerce) pour avoir déréférencé les produits d'un fournisseur par «mesure de rétorsion, qui s'analyse comme un boycott » alors qu'elle était en position dominante sur les marches des Antilles et de la Guyane (décision n97-D-20 du Conseil de la Concurrence, 25 mars 1997).
} 


\section{L'abus d'un état de dépendance économique}

L' abus de dépendance économique constitue un second type de pratique abusive, interdit par l'article L420-2 du code de commerce. Cette notion, inspirée du droit allemand, est apparue dans le droit français par les modifications apportées en 1985 à la loi Royer, relatives aux pratiques de vente discriminatoires, dont la dépendance économique constitue une circonstance aggravante. Elle est absente du droit communautaire. L'objectif poursuivi par le législateur, en introduisant ce concept dans le droit français de la concurrence, était de se doter d'un outil d'intervention dans les conflits engendrés par les rapports de force entre la grande distribution et ses fournisseurs. Cependant, cet outil ne semble pas atteindre les objectifs qui ont motivé sa création.

L'abus de dépendance économique caractérise des situations dans lesquelles une firme est un partenaire obligé d'autres entreprises auxquelles elle peut, grâce à son pouvoir de marché, imposer des conditions commerciales potentiellement anticoncurrentielles. Initialement, le texte de l'ordonnance de 1986 précisait cette notion : la dépendance économique concernait « une entreprise cliente ou fournisseur qui ne dispose pas de solution équivalente». La modification du texte par la loi sur les nouvelles régulations économiques de 2001 supprime cette référence à une "solution équivalente », laissant plus de latitude aux autorités de la concurrence pour interpréter cette définition. Notons que cette notion s'applique à des situations dans lesquelles la firme bénéficiant de la dépendance de ses partenaires n'est pas forcément en position dominante sur son marché. Le Conseil de la Concurrence précise ainsi sa définition de la dépendance économique ${ }^{20}$ : «la continuité d'exploitation au sens économique du terme de [l'entreprise] qui se trouve être en situation de dépendance n'est plus assurée, dès lors que les relations commerciales (achat ou vente de biens et de services) qu'elle entretient avec [l'entreprise dont elle est dépendante] viendraient à cesser ». Avant de sanctionner une firme pour abus d'une situation de dépendance économique, les autorités doivent prouver que la victime est bien en situation de dépendance économique, puis montrer que la firme dépendante a été victime de pratiques à caractère anticoncurrentiel.

Plusieurs critères doivent être remplis pour établir qu'une firme est en situation de dépendance économique vis-à-vis d'un fournisseur ou d'un client. La part du chiffre d'affaires réalisée avec le partenaire commercial n'est pas le seul indicateur pertinent : avant 2001, il fallait encore prouver qu'aucune «solution équivalente» n'existait. En d'autres termes, il fallait montrer qu'en cas de rupture de la relation commerciale, l'entreprise dépendante ne pouvait trouver un autre partenaire afin de conclure une nouvelle relation commerciale équivalente à la première, dans des délais et à un coût raisonnables. Ainsi, la dépendance économique naît en général de relations contractuelles de long terme, induisant des investissements spécifiques irréversibles. Dans ce cadre, le phénomène de hold-up mis en évidence par Klein, Crawford et Alkian (1978) peut alors se produire.

\footnotetext{
${ }^{20}$ Avis n ${ }^{\circ} 97-\mathrm{A}-04,21 / 01 / 1997$
} 
La dépendance économique peut a priori apparaître en amont ou en aval d'une relation verticale, selon les rapports de force au sein de la relation. La dépendance d'un distributeur à l'égard d'un fournisseur peut s'expliquer par la notoriété de la marque du produit, et la part de marché du producteur qui peut inciter le distributeur à considérer son produit comme indispensable. On parle dans ce cas de «dépendance d'assortiment». La méthodologie adoptée par le Conseil de la Concurrence pour déterminer l'existence de ce type de dépendance comporte l'étude de quatre critères : la notoriété de la marque du fournisseur, la part de marché du fournisseur pendant la période concernée par l'enquête, la part du fournisseur dans les ventes du distributeur, et enfin l'existence ou l'absence de solutions équivalentes, c'est-à-dire de produits substituts, pour le distributeur. Encore une fois, tous ces critères sont étroitement liés à la définition du marché pertinent. Dans l'ensemble, les grands distributeurs semblent peu soumis à la dépendance économique à l'égard de leurs fournisseurs. Des distributeurs puissants peuvent supporter l'absence dans leurs rayons de marques « leaders » sans se mettre en danger, comme par exemple Intermarché, qui, à la suite du conflit qui l'opposait au producteur Danone en 1997, a cessé de commercialiser plusieurs eaux minérales de renom telles que Evian, Volvic ou Badoit pendant plusieurs semaines ${ }^{21}$. Le distributeur a bien sûr perdu du chiffre d'affaires, mais sa crédibilité dans les négociations s'en est trouvée renforcée.

A l'inverse, la dépendance d'un fournisseur à l'égard d'un distributeur semble plus fréquente dans le secteur de produits de grande consommation, vendus en grandes surfaces. Le terme de « dépendance pour cause de puissance d'achat»a été créé pour refléter cette réalité. Par exemple, on évoque souvent la dépendance des producteurs de marques de distributeurs vis-àvis des distributeurs. En effet, les distributeurs exigent le plus souvent de leurs producteurs de marques propres qu'ils respectent des cahiers des charges précis, qui représentent des investissements spécifiques qui n'ont pas forcément de valeur en dehors de la relation commerciale avec ces distributeurs. Les producteurs ne sont plus véritablement libres de rompre la relation s'ils sont victimes de pratiques abusives.

La méthodologie adoptée par le Conseil de la Concurrence pour déterminer l'existence d'une dépendance économique d'un fournisseur à l'égard d'un distributeur est sensiblement différente de celle qui est utilisée pour déterminer une dépendance économique du type précédent. Deux critères sont à la base de l'analyse : la proportion du chiffre d'affaires du fournisseur réalisée avec le revendeur, et la part de marché du revendeur dans le commerce du bien concerné. En outre, le Conseil de la Concurrence peut prendre en compte la durée des relations commerciales liant les deux entreprises, ainsi que diverses caractéristiques du fournisseur comme ses ressources financières ou la notoriété de sa marque. Enfin, le Conseil

\footnotetext{
${ }^{21}$ Plusieurs conflits sont survenus entre le groupe Intermarché et ses fournisseurs durant l'année 1997. A la suite d'une campagne de publicité lancée par ce distributeur sur le thème "Changez d'air, Changez d'eau", les relations commerciales avec le groupe Danone ont été rompues. Peu de temps après la constitution de la centrale d'achat Agenor, commune à Intermarché et au distributeur allemand Spar, a amené l'enseigne française à exiger de ses fournisseurs des rabais supplémentaires de 2 à $3 \%$. A cette époque plusieurs produits de la gamme des
} 
de la Concurrence précise dans son avis n97-A-04 que « l'analyse tient également compte des raisons qui ont conduit le fournisseur à l'état de dépendance. Si celle-ci est le résultat d'un choix délibéré ayant pour objet, par exemple, d'augmenter sensiblement la production de l'entreprise au détriment de sa liberté d'action, l'entreprise ne saurait ultérieurement se prévaloir d'une option stratégique qui s'avérerait à terme mauvaise économiquement. Par contre, si l'état de dépendance résulte d'éléments conjoncturels ou structurels sur lesquels l'entreprise n'a pas de prise, la dépendance est alors subie et se pose la question de l'existence de solutions alternatives », en particulier à l'extérieur de la grande distribution.

L'analyse de la jurisprudence concernant les cas de dépendance économique d'un fournisseur à l'égard d'un distributeur montre que l'interprétation de cette notion n'est pas simple. Jusqu'à présent, le Conseil n'a jamais prononcé de condamnation à l'égard d'un groupe de la grande distribution généraliste pour abus de dépendance économique d'un de ses fournisseurs ${ }^{22}$. Ainsi, la décision n ${ }^{\circ} 93-\mathrm{D}-21 \mathrm{du}$ Conseil de la Concurrence rejette l'incrimination du groupe Cora pour abus de dépendance économique de certains fournisseurs. Le Ministère de l'Economie avait saisi le Conseil de la Concurrence de cette affaire. En 1989, le distributeur était en effet engagé dans une opération de concentration, et avait fait savoir à de nombreux fournisseurs qu'à cette occasion, il entendait renégocier ses conditions d'approvisionnement. Il avait ainsi réclamé une «participation publi-promotionnelle forfaitaire», apparemment destinée à financer l'opération de concentration, et qui lui a été effectivement versée par une cinquantaine de fournisseurs. Sans rejeter la possibilité de l'existence d'une dépendance économique de certains fournisseurs à l'égard du distributeur, le Conseil de la Concurrence a rejeté en 1993 l'accusation d'abus de dépendance économique, alléguant qu'il n'était pas possible d'établir que les pratiques du groupe Cora avaient pour effet de fausser le jeu de la concurrence à un niveau du marché. Cette décision a été confirmée par la Cour d'appel de Paris $^{23}$ en 1994.

Dans une autre affaire concernant la commercialisation des lessives en grandes surfaces ${ }^{24}$, le Conseil de la Concurrence rejette également l'allégation de dépendance économique d'un fournisseur à l'égard d'un distributeur. Sur ce marché, le secteur productif est assez concentré, puisque quatre producteurs se partagent plus de $90 \%$ des parts de marché. Il s'agit donc typiquement d'une situation équilibrée entre fournisseurs et distributeurs, en termes de concentration. Le Conseil de la Concurrence relève de nombreuses pratiques anticoncurrentielles, provenant tant des producteurs que des distributeurs, mais aucun abus de dépendance économique. La possibilité d'une dépendance économique des producteurs de

fromageries Bel ont disparus des rayons. Voir à ce sujet la presse professionnelle ("Intermarché continue à prôner l'infidélité", LSA n¹539, 05.1997, "Le bras de fer d'Agenor avec ses fournisseurs", LSA n¹592, 07.1998).

${ }^{22}$ En revanche, des condamnations à ce titre ont été rendues par les tribunaux, pour des affaires de portée très locale. Ainsi, en 1994, deux condamnations ont été prononcées par les tribunaux civils à l'encontre de magasins Leclerc pour abus de dépendance économique de fournisseurs. Dans les deux cas, le point de départ a été la suspicion de revente à perte de fruits et légumes. Suite à des plaintes déposées par les concurrents, l'enquête a révélé que les fournisseurs avaient vendu leurs produits à perte sans contrepartie réelle de la part de l'acheteur, parce qu'ils ne pouvaient résister à la puissance d'achat des centres Leclerc.

${ }_{23}$ Arrêt de la Cour d'appel de Paris du 25 mai 1994.

${ }^{24}$ Décision $n^{\circ} 94-\mathrm{D}-60$ relative à des pratiques relevées dans le secteur des lessives. 
lessives vis-à-vis d'Intermarché, qui assure environ 16,8\% des ventes de détergents en grandes et moyennes surfaces, est évoquée suite au constat de l'existence de pratiques discriminatoires avantageant ce distributeur ${ }^{25}$. Mais l'enquête fait apparaître la forte concentration du secteur des lessives et la notoriété de chacune des marques. Par ailleurs, les lessives de grandes marques sont couramment utilisées par les distributeurs comme produits d'appel* : un vaste éventail de marques est vendu avec de faibles marges et fait l'objet de promotions destinées à attirer les clients dans les magasins. Finalement, le Conseil ne retient pas l'hypothèse d'une dépendance économique des producteurs à l'égard des distributeurs : «si les relations commerciales entre les principaux producteurs de lessives et le groupement Intermarché sont fréquemment conflictuelles en raison de l'importance que revêtent pour les fabricants comme pour ce groupement de distributeurs les ventes de lessives dans cette enseigne, il ne peut être considéré que les producteurs se trouveraient en situation de dépendance économique vis-àvis du groupement Intermarché ».

Il semble finalement que la notion d'abus de dépendance économique, qui avait été créée principalement dans un objectif de régulation des relations entre industrie et commerce, ne soit pas un outil adapté. En effet, la formulation initiale de l'ordonnance de 1986 manquait de précision, engendrant ainsi des contradictions entre ce texte et d'autres principes de droit. En particulier, comme le montre Galène (1994), une interprétation trop large de la notion de solution équivalente revenait à un droit automatique de renouvellement des contrats, remettant en cause la liberté commerciale de l'un des contractants. D'autre part, malgré la situation oligopolistique du secteur de la grande distribution, les autorités de la concurrence n'ont jamais reconnu de position dominante à aucun acteur du secteur, sauf sur une zone géographique restreinte ${ }^{26}$. Ces limites expliquent que les outils juridiques créés par la définition des pratiques abusives dans l'ordonnance de 1986 aient été peu utilisés dans le cadre des relations entre producteurs et distributeurs. En définitive, la législation générale ne permettant pas aux pouvoirs publics de satisfaire une volonté politique d'intervention dans les relations entre producteurs et distributeurs, le législateur a engagé à la fin des années quatrevingt dix une réforme des règles de la concurrence afin de disposer d'un outil spécifique d'intervention sur les relations entre la grande distribution et ses fournisseurs. La loi Galland de 1996 réforme une partie de l'ordonnance de 1986 avec l'objectif affiché de protéger à la fois les fournisseurs et les concurrents de la grande distribution. Nous présentons cette loi dans la section suivante, en mettant l'accent sur ses effets directs et indirects sur les relations entre producteurs et distributeurs.

\footnotetext{
${ }^{25}$ Notons que cet argument de dépendance s'appliquerait également aux quelques très grands distributeurs qui disposent de parts de marché comparables.

${ }^{26}$ Cf note 18 concernant la décision n ${ }^{\circ 97-D-20 ~ d u ~ C o n s e i l ~ d e ~ l a ~ C o n c u r r e n c e ~ s u r ~ l e ~ c a s ~ P r i m i s t e r e s-R e y n o i r d ~ a u x ~ A n t i l l e s . ~}$
} 


\section{La loi Galland}

La loi Galland ${ }^{27}$, adoptée en 1996, réforme l'ordonnance de 1986. Son objectif est de rétablir « la loyauté et l'équilibre des relations commerciales ». Elle témoigne d'une préoccupation des pouvoirs publics relative à la puissance d'achat croissante de la grande distribution et à ses conséquences sur les relations entre fournisseurs et distributeurs, et sur la concurrence entre distributeurs. Ces deux aspects sont visés par la loi, qui a suscité un vif débat entre les différentes catégories de professionnels et les pouvoirs publics. Nous présentons d'abord les modifications majeures apportées par la loi Galland à l'ordonnance de 1986, puis nous proposons une analyse critique de leurs effets, en nous appuyant sur l'observation des comportements des entreprises depuis l'application de la loi.

\section{La transparence tarifaire et l'interdiction de la revente à perte}

Le premier volet de la réforme concerne les pratiques tarifaires, et répond directement à une plainte récurrente des producteurs. En effet, ces derniers déplorent que les prix très bas pratiqués par les distributeurs pour vendre leurs produits nuisent à leur image de marque ${ }^{28}$ auprès des consommateurs, et s'accompagnent d'une diminution de l'effort commercial des détaillants. Or le jeu de la concurrence en prix entre les distributeurs les conduit souvent à fixer les prix les plus bas possibles, en particulier pour les produits d'appel. La loi Galland propose deux «garde-fous» tarifaires : elle renforce le contrôle des offres promotionnelles des distributeurs, et précise l'interdiction de la revente à perte.

En ce qui concerne les promotions, la loi précise que lorsque « les opérations promotionnelles sont susceptibles de par leur ampleur ou leur fréquence, de désorganiser les marchés, un arrêté interministériel ou, à défaut préfectoral, fixe pour les produits concernés, la périodicité et la durée de telles opérations ». Les périodes de promotions sont donc plus encadrées qu'auparavant. Concernant la revente à perte, les modifications introduites par la loi sont plus subtiles. Les pratiques de revente à perte étaient déjà interdite par l'article 32 de l'ordonnance de 1986, qui les considère comme «déloyales » pour plusieurs raisons ${ }^{29}$. Tout d'abord, les pratiques de revente à perte peuvent être assimilées à des pratiques de prix prédateur ${ }^{30}$ visant à éliminer les petits commerces ou le commerce spécialisé. La grande majorité des cas de revente à perte ont été relevés dans les grandes surfaces alimentaires : en effet, grâce au très grand nombre de références mises en vente, les grandes surfaces alimentaires peuvent compenser les pertes réalisées sur certains produits par les gains réalisés sur d'autres, par le biais de subventions croisées entre différents types de biens. D'autre part, les pratiques de revente à perte peuvent également porter atteinte aux producteurs. En effet, lorsqu'un distributeur pratique un prix trop faible, les autres distributeurs peuvent soupçonner le

\footnotetext{
${ }^{27}$ Loi n ${ }^{\circ} 96-588$ du $1^{\text {er }}$ juillet 1996 sur la loyauté et l'équilibre des relations commerciales.

${ }^{28}$ Cet argument des producteurs est cependant discutable dans la mesure où, pour certains types de biens, des prix bas peuvent aussi être utilisés par un distributeur pour signaler la bonne qualité du produit (Cf. Tirole, 1988).

${ }^{29}$ Pour une discussion plus complète sur les motifs de l'interdiction de la revente à perte, voir Chambolle (2001).

${ }^{30}$ Voir Milgrom et Roberts (1982), et pour une analyse plus générale des stratégies de prix de détail, voir Bliss (1988).
} 
producteur de lui avoir conféré des avantages et réclamer une diminution de prix. Finalement, l'ensemble du marché intermédiaire risque de se trouver perturbé. Enfin, en revendant un produit à perte, un distributeur peut nuire à l'image de marque du produit. Cette mesure d'interdiction de la revente à perte poursuit donc à la fois un objectif horizontal, à savoir encadrer la concurrence entre les grandes surfaces et le petit commerce, et un objectif vertical puisqu'elle répond aux plaintes exprimées par les producteurs. Cependant, la formulation de l'ordonnance de 1986, et notamment la définition du seuil de revente à perte, étaient suffisamment floues pour permettre en fait aux distributeurs de contourner facilement cette interdiction. La réforme du 1er juillet 1996 clarifie la méthode de calcul du seuil de revente à perte, et renforce la sanction encourue par un commerçant en cas d'infraction.

Afin de simplifier les factures et d'y faire apparaître plus clairement le prix réellement pratiqué, les règles de facturation sont modifiées. Alors qu'une facture pouvait jusqu'alors mentionner toutes les ristournes* « dont le principe est acquis et le montant chiffrable lors de la vente $[\ldots]$ quelle que soit leur date de règlement », elle ne peut plus maintenant mentionner que les «réductions de prix acquises à la date de la vente [...] et directement liées à cette opération de vente ». En outre, le prix de référence définissant le seuil de revente à perte est fixé exactement au prix porté sur la facture d'achat majoré des diverses taxes et du prix du transport, alors que l'ordonnance de 1986 laissait une marge d'interprétation puisque le seuil était seulement "présumé être » ce prix. Ainsi, le prix d'achat effectif qui entre en ligne de compte dans le calcul du seuil de revente à perte ne peut plus intégrer les ristournes anticipées mais non encore acquises. Cette réforme empêche les distributeurs de contourner l'interdiction de la revente à perte, par exemple en fixant pour certains produits d'appel des prix de détail inférieurs aux prix de gros, et en faisant ensuite payer à leurs fournisseurs des services plus ou moins fictifs, afin de couvrir leurs frais par le biais des «marges arrière*». Les rabais* ou ristournes concédés par les producteurs, dont les principaux sont les remises* de coopération commerciale, sont couramment négociés en fin d'année, après la vente des produits concernés. Ces remises diverses pouvaient auparavant être anticipés par les distributeurs et intégrés notamment dans le calcul du seuil de revente à perte. Elles doivent maintenant être facturées et ne peuvent être prises en compte qu'une fois qu'elles ont été effectivement versées ${ }^{31}$. En outre, les services commerciaux fournis par le distributeur, comme la disposition des articles sur des têtes de gondoles* ou les diverses activités promotionnelles proposées par les distributeurs (animation des rayons, publication de catalogues envoyés chez les consommateurs) doivent eux aussi faire l'objet d'une facturation séparée. En conséquence, les marges arrière ne peuvent plus jouer le rôle qu'elles tenaient auparavant dans les négociations entre producteurs et distributeurs pour contourner l'interdiction de la revente à perte.

En outre, une exemption à l'interdiction de la revente à perte existait auparavant, afin de protéger la concurrence par les prix des variations des conditions d'approvisionnement: le

\footnotetext{
${ }^{31}$ Bien entendu, les ristournes acquises une année ne peuvent être répercutées sur le prix de vente du même bien, l'année suivante, puisque seules peuvent l'être les ristournes « directement liées à [1'] opération de vente » du bien concerné par la facture.
} 
«droit d'alignement » permettait ainsi à un distributeur de vendre un produit à perte pour s'aligner sur les tarifs d'un concurrent direct, situé dans la même zone de chalandise. La loi Galland supprime ce droit d'alignement, sauf pour les magasins alimentaires de moins de 300 $\mathrm{m}^{2}$ et non-alimentaires de moins de $1000 \mathrm{~m}^{2}$. Elle donne ainsi, du moins théoriquement, un droit supplémentaire au commerce de proximité.

\section{L'interdiction des prix abusivement bas}

Les « prix abusivement bas » sont prohibés par le nouvel article 10-1. Mais aucun seuil n'est clairement défini, et le terme d'abusivement bas, qui s'entend apparemment par rapport aux coûts de production et de commercialisation, n'est pas précisé. Cette clause concerne les produits transformés vendus directement aux consommateurs, mais pas les produits revendus en l'état qui sont protégés par l'interdiction de la revente à perte. L'exemple de la « baguette à un franc ", vendue sous marque de distributeur par une grande enseigne, semble avoir été à l'origine de ce nouvel article : les producteurs et les distributeurs concurrents ne pouvaient faire face à ce prix prédateur, mais il était impossible de montrer que le bien était revendu à perte, car le fabricant du pain était intégré verticalement au distributeur. Cette clause protège ainsi indirectement les fournisseurs de marques nationales d'une concurrence trop vive des marques de distributeurs.

\section{L'autorisation du refus de vente}

Le second volet de la réforme concerne les conditions du référencement, et vise à donner dans ce domaine plus de pouvoir aux producteurs et moins aux distributeurs. La circulaire Fontanet du 31 mars 1960, qui interdisait le refus de vente, est abrogée. Les producteurs peuvent donc désormais refuser de fournir certains distributeurs, sans explication, sauf dans le cas où ils occupent une position dominante. Cependant, les producteurs restent soumis à l'interdiction de proposer des conditions commerciales discriminatoires à certains de leurs clients. On verra plus loin que, si le respect de cette règle au niveau des conditions générales de vente (qui sont toujours publiques) est relativement facile à contrôler, il l'est beaucoup moins au niveau des marges arrière. Enfin, le dernier point de la loi précise que les producteurs déjà en relation avec un distributeur ne peuvent rompre sans préavis la relation commerciale, ce qui exclut un refus de vente brutal et sans préavis d'un fournisseur habituel.

L'autorisation du refus de vente vise principalement les magasins de maxidiscompte*. De nombreux producteurs se plaignaient en effet d'être obligés de vendre leurs produits à ces distributeurs, qui ne fournissent qu'un effort de vente restreint, et ne mettent pas en valeur les produits qu'ils commercialisent, afin de réduire le plus possible leurs coûts et de proposer des prix très bas. D'après ces producteurs, ce type de commercialisation nuit à l'image de marque, et donc indirectement à la valeur de leurs produits. L'interdiction du refus de vente les empêchait de s'opposer à la commercialisation de leurs produits dans ce type de commerces, et favorisait ainsi le développement du maxidiscompte à leurs dépens. 


\section{La réglementation des primes de référencement}

La loi Galland prohibe les primes de référencement versées sans contreparties réelles : il est désormais interdit «d'obtenir ou de tenter d'obtenir un avantage, condition préalable à la passation de commandes, sans l'assortir d'un engagement écrit sur un volume d'achat proportionné et, le cas échéant, d'un service demandé par le fournisseur et ayant fait l'objet d'un accord écrit ». Ainsi, la pratique courante des primes de référencement est réglementée, et la nouvelle loi interdit certains abus constatés auparavant. En particulier, un producteur qui paie une prime de référencement est assuré qu'une certaine quantité de ses produits va être commercialisée : les distributeurs demandant des primes de référencement doivent maintenant s'engager clairement à acheter une quantité minimale qui doit être proportionnelle à la prime. Cependant, encore une fois, aucun seuil précis n'est défini, et la loi reste ambiguë.

\section{L'encadrement juridique du déréférencement}

Enfin, le «déréférencement abusif» est prohibé. L'article 36 de l'ordonnance de 1986 est modifié, et le nouveau texte interdit «de rompre brutalement, même partiellement, une relation commerciale établie, sans préavis tenant compte des relations commerciales antérieures ou des usages reconnus par des accords interprofessionnels ». Toute rupture d'une relation commerciale doit donc désormais s'accompagner d'un préavis écrit. Cette disposition s'applique aussi aux producteurs souhaitant rompre l'approvisionnement d'un distributeur. En outre, les menaces de déréférencement utilisées pour obtenir des conditions commerciales discriminatoires sont prohibées : il n'est pas autorisé « d'obtenir ou de tenter d'obtenir, sous la menace d'une rupture brutale des relations commerciales, des prix, des délais de paiement, des modalités de vente ou des conditions de coopération commerciale manifestement dérogatoires aux conditions générales de vente ». Mais cette mesure qui vise à interdire le chantage au déréférencement lors des négociations entre producteurs et distributeurs est avant tout une affirmation de principe, et une telle interdiction est très difficilement applicable.

\section{Les effets de la loi Galland}

L'énoncé des différentes modifications apportées à la législation par la loi Galland a permis de souligner certaines de ses limites. Nous cherchons maintenant à évaluer ses conséquences réelles sur les relations entre producteurs et distributeurs. En effet, la loi Galland a de nombreux effets pervers ${ }^{32}$, dus pour la plupart à l'ambiguïté de sa formulation. Le texte est particulièrement flou sur un certain nombre de points, ce qui en rend difficile l'application. Ainsi, aucun seuil précis n'est défini dans le texte de la loi, ni pour définir ce qu'est un prix « abusivement bas », ni pour établir quel volume d'achat doit être garanti lors du versement d'une prime de référencement. En outre, l'interdiction des menaces de déréférencement se heurte à la difficulté de produire des preuves, les menaces de ce type étant en général

\footnotetext{
${ }^{32}$ Voir Caprice et Philippe (1997).
} 
informelles et rarement écrites. Enfin, la plupart des nouvelles règles peuvent être contournées par les firmes.

Tout d'abord, certaines mesures peuvent avoir un effet légèrement positif pour les producteurs. Ainsi, l'interdiction de revente à perte peut être comprise comme un moyen détourné pour le législateur d'autoriser la pratique des prix de revente imposés. En effet, un producteur peut choisir de facturer au distributeur le prix, étant donné les conditions de concurrence du marché, auquel il souhaite que son produit soit revendu. Le distributeur, n'ayant pas le droit de revendre ce produit à perte, est obligé de respecter le prix minimum ainsi imposé par le producteur, et ce dernier peut rétribuer le distributeur par le biais des marges arrière. Cette réforme traduit un choix délibéré du législateur d'intervenir en faveur des producteurs. Cependant, il semble que cette mesure soit déjà contournée par les grands distributeurs, qui parviennent à négocier l'intégration progressive des remises de fin d'année dans les factures en cours d'année. D'autre part, en déplaçant le champ de la négociation entre producteurs et distributeurs des marges avant vers les marges arrière, la loi ne donne aucun outil de lutte contre les pratiques discriminatoires, pourtant théoriquement interdites : si les producteurs sont contraints de proposer à tous leurs clients les mêmes conditions générales de vente, ils sont en revanche nettement plus libres de fixer à leur guise le montant des marges arrière, par exemple en rémunérant des services de distribution dont il est difficile d'évaluer l'influence réelle sur les ventes. Ces conditions sont généralement négociées secrètement, et il est très difficile pour les autorités de la concurrence d'estimer si elles sont justifiées ou si elles expriment simplement le pouvoir de négociation du distributeur.

Ensuite, les distributeurs pourraient se trouver également avantagés par cette mesure d'interdiction de la revente à perte puisque ce mécanisme de prix imposé limite directement la guerre des prix.

Par ailleurs, comme l'interdiction de la revente à perte n'a pas été adoptée par les autres pays européens, des écarts de prix importants peuvent survenir. A titre d'exemple, le distributeur Leclerc a déclaré avoir vendu des bouteilles d'Orangina au prix de 5,95 $\mathrm{F}$ en Belgique tandis que le prix sur le marché français était de $7,50 \mathrm{~F}^{33}$. En effet, le seuil de revente à perte tel qu'il est fixé en France interdisait à Leclerc de revendre le produit à un prix plus faible. Comme d'autres producteurs, Orangina fixe des prix unitaires élevés, et négocie des primes de coopération commerciale avec ses distributeurs : ces primes, auparavant marginales, peuvent atteindre depuis la loi Galland $45 \%$ du prix de gros $^{34}$.

En ce qui concerne l'autorisation du refus de vente, cette mesure pourra permettre à certains producteurs de choisir leur circuit de distribution, et en particulier de ne pas approvisionner les magasins de maxidiscompte. Mais on peut attendre aussi des effets pervers de cette

\footnotetext{
${ }^{33}$ LSA, 30/09/1999.

${ }^{34}$ Questions parlementaires, 2000-27.
} 
nouvelle mesure. Ainsi, elle risque d'engendrer des pratiques de forclusion ${ }^{35 *}$, car un distributeur disposant d'un fort pouvoir de négociation peut exiger d'un fournisseur qu'il cesse d'approvisionner un distributeur concurrent. Le producteur ne peut pas se retrancher derrière l'interdiction du refus de vente, et, comme on l'a vu plus haut, la notion d'abus de dépendance économique s'appliquant rarement aux relations entre producteurs et distributeurs, il est mal protégé par la loi dans une telle situation. Les mesures concernant le préavis de déréférencement peuvent elles aussi être contournées : ces mesures risquent d'aboutir à la suppression partielle des contrats de long terme, remplacés par une succession de contrats de court terme ou « spots ». Cela risque ainsi en fait de fragiliser les relations contractuelles entre les distributeurs et leurs petits fournisseurs.

Un autre défaut majeur du nouveau texte est qu'il confond puissance d'achat et taille des magasins. Les magasins de moins de $300 \mathrm{~m}^{2}$ peuvent appartenir à un distributeur qui dispose d'une très forte puissance d'achat. De nombreux magasins de maxidiscompte en particulier occupent une surface inférieure à cette limite, de même que certaines chaînes de distribution alimentaire en centre-ville. La loi traite ces magasins, qui bénéficient d'une forte puissance d'achat grâce aux centrales d'achat auxquelles ils sont affiliés, comme les petits commerces indépendants. De ce fait, elle manque un de ses objectifs, qui était de donner au «petit commerce » des armes pour lutter contre la grande distribution.

En outre, la loi Galland a eu à court terme un effet inflationniste. D'après une étude réalisée par IRI-Sécodip sur un panier de produits de consommation courante, les prix ont augmenté dans les grandes surfaces de 1,5\%, hors inflation, sur le premier trimestre de l'année 1997. En fait, cet effet est sans doute dû à la diminution sensible du nombre de reventes à perte. Enfin, la loi Galland semble avoir contribué à accélérer le développement des marques de distributeurs, les grands groupes de distribution ayant souvent choisi cette voie pour réagir aux nouvelles contraintes que leur imposaient les pouvoirs publics. Ainsi, une étude réalisée en 1997 par SECODIP fait apparaitre que dans les premiers mois qui ont suivi la mise en œuvre de la loi Galland, les parts de marchés des marques de distributeurs se sont accrues de 1 à 3 points selon les marchés ${ }^{36}$. De surcroît, le groupement Leclerc, qui était pourtant resté jusqu'alors hostile à cette évolution, a beaucoup investi dans ses marques propres depuis 1996, en créant sa «marque repère ». L'idée selon laquelle la loi Galland aurait nettement pesé dans la décision de Leclerc a été avancé à l'époque dans la presse professionnelle ${ }^{37}$.

En conclusion, la loi Galland a eu des effets mitigés. Très contestée par les professionnels de la distribution, elle a été accueillie avec retenue et parfois même avec méfiance par les diverses catégories de producteurs. Dans l'ensemble, ses moyens ne semblent pas avoir été à la hauteur de ses objectifs, et, depuis son adoption en 1996, la loi n'a pas fondamentalement transformé le paysage des relations entre producteurs et distributeurs en France. Dans la

\footnotetext{
${ }^{35}$ Voir Ordover, Saloner et Salop (1990).

${ }^{36}$ Voir "les MDD dopées par la loi Galland", Linéaires n¹18, 09.1997.

37 "Pourquoi Leclerc repère ses MDD...", Linéaires, n¹15, 05.1997.
} 
section suivante, nous présentons la réglementation des structures du marché de la distribution, et en particulier les règles de contrôle des concentrations et la loi Raffarin, qui est le second volet des modifications réglementaires adoptées en 1996.

\section{Le contrôle des structures}

Afin de réguler les relations entre industrie et commerce, les pouvoirs publics exercent également un contrôle sur les structures de marché. En effet, la structure d'un marché est déterminante non seulement pour la concurrence horizontale entre les firmes du secteur, mais également dans les relations qu'elles entretiennent avec leurs partenaires verticaux. Ainsi, la concentration du secteur de la distribution est un point central du débat sur les rapports de force entre producteurs et distributeurs : comme on l'a vu plus haut, le récent mouvement de concentration dans la distribution a fortement accru la puissance d'achat des grands distributeurs. Le taux de concentration de la grande distribution a augmenté nettement plus ces dernières années que celui de la plupart de ses fournisseurs ${ }^{38}$. Or l'augmentation de cette puissance d'achat peut restreindre le jeu de la concurrence en modifiant les conditions d'entrée et de survie des producteurs sur le marché amont. La concentration des distributeurs agit à deux niveaux sur les fournisseurs : d'une part, un seul partenaire (le distributeur résultant de la fusion) leur donne accès à une part de marché final plus importante, ce qui réduit les coûts de transaction, mais d'autre part la concentration réduit le nombre de leurs clients potentiels. Les distributeurs peuvent ainsi négocier de meilleures conditions d'achat, et leur concentration peut mettre en danger certains petits producteurs, en les plaçant en situation de dépendance économique vis-à-vis de leurs clients, et en menaçant leur survie à moyen terme.

\section{Le contrôle des concentrations dans la distribution}

D’une manière générale, les opérations de concentration entre entreprises françaises font l'objet d'un contrôle au niveau communautaire si les groupes concernés réalisent une grande part de leur chiffre d'affaires dans d'autres pays de la Communauté Européenne. Les opérations peuvent ensuite être renvoyées partiellement ou totalement aux autorités nationales, chargées en général de mesurer leur impact sur les marchés nationaux. En France, producteurs et distributeurs sont soumis aux mêmes règles du contrôle des concentrations, mais depuis les années quatre-vingt-dix les autorités en charge de ce contrôle se sont interrogées à plusieurs reprises sur la nécessité de créer des règles spécifiques à la distribution, dans la mesure où ce secteur d'activité connaissait un mouvement de concentration fort et rapide. Dans ce secteur particulièrement, il est nécessaire d'évaluer les conséquences des fusions aussi bien sur les marchés de détail que d'approvisionnement. Nous présentons rapidement l'objectif du contrôle des concentrations puis nous précisons le processus de contrôle spécifiquement adapté au secteur de la grande distribution. Nous

\footnotetext{
${ }^{38}$ Voir Charléty et Souam (2000).
} 
analysons ensuite son influence sur les relations entre fournisseurs et distributeurs, et nous concluons par un rapide bilan.

\section{Objectifs et méthodologie du contrôle}

L'objectif du contrôle des concentrations est de prévenir les fusions qui pourraient s'avérer économiquement néfastes ${ }^{39}$, c'est-à-dire qui pourraient «porter atteinte à la concurrence, notamment par création ou renforcement d'une position dominante ». Le texte de l'ordonnance de 1986, très général, ne mentionne pas d'autres facteurs à prendre en compte. L'avis n97-A-04 du Conseil de la Concurrence en date du 21 janvier 1997 est consacré plus précisément à la définition d'une méthodologie pour le contrôle des concentrations dans la grande distribution. Cette méthode comporte trois étapes. La première est la définition du marché pertinent, le « lieu théorique où se confrontent l'offre et la demande de produits ou de services qui sont considérés par les acheteurs ou les utilisateurs comme substituables entre eux mais non substituables aux autres biens ou services offerts ». Nous avons déjà évoqué l'importance de cette délimitation dans le cadre de la législation sur les abus de position dominante.

La deuxième étape du contrôle d'une concentration est la prise en compte des critères de parts de marchés. Seules sont soumises à l'autorisation du ministère en charge de l'économie les opérations de concentration entre entreprises qui « ont soit réalisé ensemble plus de $25 \%$ des ventes, achats ou autres transactions sur un marché national de biens [...] substituables ou sur une partie substantielle d'un tel marché, soit totalisé un chiffre d'affaires hors taxes de plus de sept milliards de francs, à condition que deux au moins des entreprises parties à la concentration aient réalisé un chiffre d'affaires ${ }^{40}$ d'au moins deux milliards de francs ». Si ces conditions sont respectées, le ministère de l'économie peut demander au Conseil de la Concurrence un avis, qui peut ensuite être suivi ou rejeté. Dans certains cas, le Conseil peut demander que soient prises des mesures correctives, comme celles qu'il a préconisées lors de la récente opération de concentration entre Carrefour ${ }^{41}$ et Promodès, ou lors de l'OPA d'Auchan sur Docks de France en $1996^{42}$.

La troisième étape est l'analyse des conséquences concurrentielles à la fois sur le marché de détail et sur le marché de l'approvisionnement. Le bilan concurrentiel tient compte des gains dus à d'éventuelles économies d'échelle ou de gamme. En aval, les atteintes à la concurrence sur le marché de détail sont essentiellement l'accroissement des prix à la consommation, dû à

\footnotetext{
${ }^{39}$ Pour une analyse de l'impact des fusions sur le surplus social, voir Farrell et Shapiro (1990), Kamien et Zang (1993) et Werden et Froeb (1994).

${ }^{40} \mathrm{La}$ loi sur les nouvelles régulations économiques du 15 mai 2001 modifie ces seuils à 150 millions d'euros pour le premier et 15 millions d'euros pour le second.

${ }^{41}$ Cette opération a d'abord reçu l'aval de la Commission européenne, sous condition que le groupe Carrefour revende ses $41 \%$ de parts détenues dans le groupe Cora à un ou plusieurs concurrents indépendants. La Commission a ensuite renvoyé l'affaire devant les autorités nationales pour qu'elles contrôlent l'influence de l'opération sur les marchés locaux. Finalement, les autorités françaises ont exigé de Carrefour qu'il s'engage à revendre certains de ses hypermarchés situés dans des zones où la concentration pouvait aboutir à la constitution d'une position dominante locale.

${ }^{42}$ Avis n $96-A-11$ du 10 septembre 1996 relatif à la prise de participation, suivie d'une offre publique d'achat, du capital de la société Docks De France, réalisée par la société Auchan. L’avis préconise des mesures correctives à Strasbourg, Aubagne et Perpignan.
} 
l'augmentation du pouvoir de marché des distributeurs, et la diminution de la variété des produits proposés qui nuit aux consommateurs. Le contrôle doit également prendre en compte l'influence de l'opération en amont, sur les relations entre fournisseurs et distributeurs. Une concentration peut, en théorie, être refusée si elle renforce trop la puissance d'achat des distributeurs. Ainsi, cet avis précise les facteurs à prendre en compte dans l'appréciation du bilan concurrentiel des opérations de concentration, qui ne figuraient pas dans le texte de l'ordonnance, et notamment tout ce qui concerne les effets verticaux.

En effet, le contrôle des concentrations est le seul moyen à la disposition des pouvoirs publics pour empêcher ex ante la formation ${ }^{43}$ d'une position dominante, sans qu'il soit nécessaire de prouver l'existence d'un comportement abusif ou de pratiques anticoncurrentielles. Dans le contexte actuel des relations entre la grande distribution et ses fournisseurs, on comprend l'intérêt que présente cet outil de régulation. Cependant, jusqu'à présent, les autorités de la concurrence ont peu utilisé cet outil, jugeant que la grande distribution n'occupe pas une position structurellement dominante. Ainsi, le Conseil de la Concurrence exprime dans son avis sur l'opération de rachat des enseignes Leader Price et Franprix par Casino en 1999, l'opinion que même si l'opération fait disparaître un distributeur indépendant et renforce la position de Casino, il n'y a pas lieu de parler de position dominante sur le marché de l'approvisionnement. Toutefois, l'avis « ne préjuge pas des suites à donner à un éventuel contentieux fondé sur des pratiques de déréférencement abusif ${ }^{44}$. L'analyse menée lors du rachat de Docks de France par Auchan en 1996 est plus nuancée, mais la conclusion est identique. Le Conseil reconnaît que cette concentration peut conduire à des déréférencements, mais précise que la loi Galland suffit à contrôler les pratiques abusives qui risquent de survenir.

Notons qu'un vide juridique entoure la constitution de centrales d'achat, souvent considérée comme la création d'une filiale commune. La législation nationale comme la législation communautaire hésitent à ce sujet (comme en ce qui concerne toutes les formations de « joint ventures ») entre deux régimes: celui des concentrations et celui des ententes. La Commission a publié en 1990 une notice sur la distinction entre les ententes coopératives et concentratives qui sert de référence pour le moment, mais aucun texte français n'établit clairement la démarche à suivre pour les autorités de la concurrence face à un tel cas ${ }^{45}$.

\section{Bilan des opérations de contrôle de la distribution}

Dans son avis de 1997 relatif à diverses questions portant sur la concentration du secteur de la distribution, le Conseil de la Concurrence mesure le degré de concentration atteint en France dans ce secteur, et en évalue les conséquences tant sur la concurrence en aval que sur les

\footnotetext{
${ }^{43}$ L'article 43 de l'ordonnance de 1986 précise même que le Conseil de la Concurrence peut demander au ministre de revenir sur une autorisation de concentration accordée, s'il s'avère que cette concentration a entraîné un abus de position dominante.

${ }^{44}$ Avis n98-A-06 du Conseil de la Concurrence en date du 5 mai 1998 relatif à l'acquisition par la société Casino-Guichard-Perrachon de la société TCL Béatrice Holdings France SA (enseignes Franprix-Leader Price).

${ }^{45}$ A ce sujet, on consultera AFEC (1999) et Lang (2000).
} 
relations entre fournisseurs et distributeurs. Il préconise, dans le cadre du contrôle d'une opération de concentration entre distributeurs, de comparer la puissance d'achat des distributeurs au pouvoir de marché des producteurs, afin d'en déduire les risques de dépendance économique. Cette analyse ne peut donc être menée qu'au cas par cas, et le Conseil écarte toute mesure générale de restriction de la concentration au sein du secteur distributif : «aucune mesure de nature structurelle ne peut être prise de façon systématique, dès lors qu'il n'existe pas d'éléments permettant d'établir l'existence d'atteintes à la concurrence sur un ou plusieurs marchés définis. En particulier, aucune mesure de déconcentration des structures commerciales ne paraît pouvoir être fondée sur une approche générale. »

Les autorités françaises en charge de la politique de la concurrence ne sont donc pas disposées à freiner le mouvement de concentration de la grande distribution ou à s'opposer à la poursuite de son développement. La totalité des opérations de concentration entre distributeurs ont jusqu'ici été acceptées en France, même si la plupart ont donné lieu à l'imposition de mesures correctives. Les prises de participation minoritaires ont également été autorisées ${ }^{46}$. Cependant, dans d'autres pays, les autorités en charge de la concurrence sont nettement plus réticentes à accepter les propositions de concentration en provenance du secteur de la distribution. Ainsi, les autorités anti-trust américaines sont beaucoup plus sévères, et elles utilisent notamment une définition du marché pertinent plus étroite, empêchant certains projets de fusion d'être acceptés $^{47}$. La Commission Européenne a généralement adopté une attitude conciliante à l'égard des fusions dans le secteur de la distribution. Cependant, elle a récemment refusé certains projets de concentration entre distributeurs, jugeant que dans certains cas, le degré de concentration atteint était suffisant, et que la poursuite d'un processus de concentration par fusions ou acquisitions pouvait être dommageable à l'économie. Ainsi, en 1996, l'opération de concentration entre les groupes finlandais Kesko et Tuko a été jugée inacceptable, en raison de la position dominante qu'elle conférait au nouveau groupe sur le marché finlandais (plus de $60 \%$ des ventes au détail). En outre, elle a aussi imposé des conditions restrictives à certains projets de fusion, comme celui de Rewe et Reinl ${ }^{48}$ en 1999. Ces refus dénotent un changement d'attitude de la Commission à l'égard du secteur de la distribution et du problème de la puissance d'achat.

\section{De la loi Royer à la loi Raffarin : la régulation des implantations commerciales.}

Le contrôle des structures de marché passe également en France par la surveillance des implantations commerciales. Dès le début des années 1970, l'encadrement des circuits de distribution évolue vers un contrôle plus ferme du développement des grandes surfaces. En

\footnotetext{
${ }^{46}$ Par exemple la prise de participation de Carrefour dans le capital de GMB, société exploitant l'enseigne Cora, en 1997 (Avis n $97-A-14$ ).

${ }^{47}$ On peut évoquer le cas ancien mais représentatif de Von's, où la Federal Trade Commission a interdit la fusion de deux distributeurs de Los Angeles, dont les parts de marché locales cumulées ne dépassaient pas 7,5\%, pour la simple raison que le taux de concentration du secteur augmentait, et sans analyser les conséquences concurrentielles de cette concentration (1966, United States vs. Von's Grocery Company, 384 US 270).

${ }^{48}$ Décision du 03 février 1999, M 1221.
} 
1973, la loi d'orientation du commerce et de l'artisanat ${ }^{49}$, dite loi Royer, limite l'installation de nouvelles grandes surfaces. L'objectif de la loi est clairement affiché dans son article 1 : « Les pouvoirs publics veillent à ce que l'essor du commerce [...] permette l'expansion de toutes les formes d'entreprises, indépendantes, groupées ou intégrées, en évitant qu'une croissance désordonnée des formes nouvelles de distribution ne provoque l'écrasement de la petite entreprise et le gaspillage des équipements commerciaux ». Il s'agit donc de protéger le petit commerce, en évitant un développement trop rapide de la grande distribution. Nous verrons que cette mesure a également des effets non négligeables sur les relations entre producteurs et distributeurs.

Le moyen proposé par la loi Royer pour limiter le développement de la grande distribution est de restreindre les implantations de nouvelles grandes surfaces. L'installation d'entreprises commerciales au-delà d'une certaine surface $\left(1000 \mathrm{~m}^{2}\right.$ de surface de vente pour les communes de moins de 40000 habitants, $1500 \mathrm{~m}^{2}$ pour les autres communes) ainsi que certaines extensions de magasins sont soumises à l'approbation des Commissions Départementales d'Urbanisme Commercial. La loi Royer freine nettement l'implantation des très grandes surfaces : entre 1974 et 1992, les interdictions de création portent sur 23 millions de $\mathrm{m}^{2}$ de surface commerciale, alors que 16 millions de $\mathrm{m}^{2}$ sont acceptés. Mais de nombreuses affaires de corruption entachent le fonctionnement des commissions chargées d'accorder les autorisations, et la loi doit être modifiée. En 1993, la loi Sapin ${ }^{50}$ modifie la composition des Commissions, et le gouvernement annonce des suspensions provisoires des autorisations d'ouvertures de grandes surfaces. Diverses réformes rendent progressivement plus restrictives les conditions d'autorisation des nouvelles installations.

Cette évolution progressive aboutit tout d'abord, en février 1996, à une décision ministérielle de geler jusqu'à la fin de l'année les ouvertures de magasins de plus de $300 \mathrm{~m}^{2}$, puis au vote de la loi n96-603 du 5 juillet 1996 relative au développement et à la promotion du commerce et de l'artisanat, dite loi Raffarin. Cette loi apporte les modifications suivantes à la loi Royer : le seuil au-delà duquel l'autorisation des commissions est nécessaire est abaissé à $300 \mathrm{~m}^{2}$, et pour la création de magasins d'une surface supérieure à $6000 \mathrm{~m}^{2}$, une « enquête publique portant sur les aspects économiques, sociaux et d'aménagement du territoire du projet » est nécessaire. Après avoir précisé les fonctions du commerce et de l'artisanat, et notamment le rôle qu'ils doivent avoir dans le développement économique, la loi annonce un programme « de développement et de modernisation des activités commerciales et artisanales », qui doit être publié par le gouvernement. Ce programme semble cependant rapidement oublié.

La loi Raffarin reflète une volonté politique de juguler le développement de la grande distribution, et en particulier des nouveaux magasins de maxidiscompte, pour deux raisons majeures : protéger d'une part les petits producteurs, et d'autre part le « petit commerce» ou

\footnotetext{
${ }^{49}$ Loi n ${ }^{\circ} 73-1193$ du 27 décembre 1973 d'orientation du commerce et de l'artisanat.

${ }^{50}$ Loi du 29 janvier 1993.
} 
commerce de proximité, en limitant les implantations de grandes surfaces. Finalement, en 1997, 25\% des projets d'ouverture ont été rejetés par les commissions et depuis 1996 le nombre d'ouverture de supermarchés a significativement diminué (moins de 200 par an, alors qu'il y en avait plus de 300 par an entre 1986 et 1995).

On peut reprocher à la loi Raffarin d'engendrer de nombreux effets pervers. L'un des défauts majeurs de ce texte est qu'il confond, comme la loi Galland, puissance d'achat et taille des magasins : le seuil assez faible de $300 \mathrm{~m}^{2}$ touche de petits distributeurs, les empêchant de développer des magasins de libre service de taille relativement faible ${ }^{51}$. De plus, en stoppant les possibilités de croissance interne, la loi incite les distributeurs à recourir à la croissance externe, et explique une partie du mouvement actuel de concentration dans le secteur. Mais surtout, cette loi met en place, de fait, des barrières à l'entrée sur un secteur déjà oligopolistique. La limitation de l'entrée par la loi Raffarin engendre une limitation artificielle de la concurrence, et renforce ainsi la puissance des distributeurs. Cet effet a été immédiatement perçu par le marché, puisqu'à l'annonce de la loi, les cours des actions des grands distributeurs cotés en bourse ont connu une nette augmentation par rapport au CAC 40. En limitant le développement de la surface de vente totale, la loi a entraîné une revalorisation des surfaces acquises, renforçant de ce fait la puissance financière des grands groupes. En outre, cette mesure renforce le pouvoir de négociation des distributeurs face à leurs fournisseurs : cette instauration de barrières à l'entrée institutionnelles, jointe à la restriction des extensions de magasins existants, limite le linéaire*, que l'on assimile parfois à une ressource rare ${ }^{52}$. La pression concurrentielle sur les fournisseurs, qui sont en concurrence pour l'accès au linéaire, est de ce fait renforcée, d'autant plus que les producteurs doivent maintenant faire face à la concurrence des marques de distributeurs. En conséquence, cette mesure risque d'accentuer la dépendance des producteurs à l'égard des distributeurs, et de nuire à l'équilibre des relations entre industrie et commerce. Globalement, la loi Raffarin semble plutôt avoir été profitable aux grands groupes de distribution, ce qui va clairement à l'encontre de ses objectifs affichés.

\section{Conclusion}

Depuis l'ordonnance de 1986 qui a posé les principes de la libéralisation de l'économie et le cadre de sa régulation, la réglementation a évolué pour suivre les modifications des relations commerciales entre les grands distributeurs et leurs fournisseurs. Dix ans après l'élaboration des principes généraux du droit de la concurrence français, les pouvoirs publics, constatant l'inversion du rapport de forces entre producteurs et distributeurs, ont élaboré des réformes visant à instaurer une réglementation spécifique au secteur de la grande distribution et à ses

\footnotetext{
${ }^{51} \mathrm{Du}$ point de vue de l'aménagement du territoire, on peut en outre noter que la situation de référence du texte est celle des périphéries urbaines, mais la loi n'est pas adaptée aux zones rurales, où l'installation d'une grande surface est souvent considérée comme bénéfique au développement du petit commerce. En effet, dans les zones rurales, l'activité commerciale se concentre souvent autour de pôles organisés autour d'une grande surface.

${ }^{52}$ Voir à ce sujet Philippe (1997) et Allain et Flochel (2001).
} 
relations avec ses fournisseurs. Les modifications du cadre juridique qui ont résulté de cette démarche, essentiellement dues aux lois Galland et Raffarin, sont encore contestées par une partie des professionnels plusieurs années après leur mise en application. Nous avons évoqué dans cet article les ambiguïtés de ces lois et leurs effets pervers, qui nuisent à leur efficacité. En outre, en dépit des protestations des fournisseurs qui font connaître les pressions imposées par les distributeurs, très peu de plaintes sont déposées au titre de la dépendance économique. La question qui se pose est donc de savoir si la notion de dépendance économique telle qu'elle est définie par les autorités n'est pas trop large, et plus généralement si l'intervention publique est bien adaptée pour répondre au déséquilibre du rapport de force dans les relations commerciales.

Devant ce constat d'échec partiel, les pouvoirs publics ont entamé de nouvelles réflexions sur les modifications à apporter à la réglementation pour améliorer son efficience. Afin de consulter un échantillon assez vaste des professionnels des différents secteurs concernés par le développement de la grande distribution, le gouvernement a organisé les Assises de la Distribution le 13 janvier 2000. Divers travaux préparatoires, dont le rapport sur l'évolution de la distribution rédigé par la Commission de la production et des échanges de l'Assemblée Nationale, ont également alimenté la réflexion des pouvoirs publics, qui a abouti à l'élaboration de la loi relative aux nouvelles régulations économiques. Cette loi comporte trois parties, et la deuxième partie est spécifiquement consacrée à la régulation de la concurrence.

Le premier titre de la deuxième partie est consacré à la «moralisation des pratiques commerciales ». Ses objectifs, clairement affichés dans le projet de loi, sont les suivants :

«La concentration de la distribution et les dérives de comportement dans les relations entre fournisseurs et distributeurs créent le besoin d'une régulation commerciale adaptée à ce nouvel environnement [...]. Les mesures proposées ont pour objet en premier lieu de favoriser le développement de meilleures pratiques commerciales entre fournisseurs et distributeurs et de mieux identifier les pratiques abusives, ce que permettra notamment la création d'une commission des pratiques commerciales et des relations entre fournisseurs et distributeurs. »

Pour atteindre ces objectifs, la loi propose plusieurs moyens. Tout d'abord, les marchés agricoles bénéficient d'un règlement d'exemption particulier autorisant des accords entre producteurs, et notamment, dans certaines limites, des prix concertés (article L420-4). De même, concernant le marché très sensible des fruits et légumes frais, le texte amende l'ordonnance de 1986 en autorisant des accords interprofessionnels interdisant, pour des durées n'excédant pas trois mois, les opérations promotionnelles, "pour faire face aux crises conjoncturelles ». Toutefois, cette mesure risque de manquer son objectif dans la mesure où en situation de surproduction, les distributeurs pourront continuer de faire pression sur les producteurs pour s'approvisionner au prix le plus bas sans pour autant répercuter cet avantage 
sur le prix aux consommateurs. En outre, une Commission des pratiques commerciales ${ }^{53}$ est créée afin d'observer l'évolution des relations contractuelles entre fournisseurs et distributeurs. Enfin, le nouveau texte précise différentes notions qui restaient floues. En particulier, il établit que toute coopération commerciale ou marge arrière sans contrepartie réelle est présumée constituer un avantage discriminatoire. De plus, le fait d'user de menaces de déréférencement partiel (c'est-à-dire portant sur une partie de la gamme de produits du fournisseur) expose aux mêmes sanctions que les menaces de déréférencement total. Le texte prévoit également que la durée du préavis de déréférencement, qui n'est pas précisée dans la loi Galland, doit être publiée dans les accords interprofessionnels, et peut être imposée dans certains cas par arrêté ministériel.

Par ailleurs, le titre II de la deuxième partie propose d'améliorer les moyens mis à la disposition des autorités pour lutter contre les pratiques anticoncurrentielles, en prévoyant notamment un alourdissement des sanctions pouvant être infligées aux entreprises en faute par le Conseil de la Concurrence. Enfin, la réglementation sur le contrôle des concentrations est également modifiée. En particulier, la nouvelle loi préconise d'examiner si l'opération de concentration est de nature à «porter atteinte à la concurrence, notamment par création ou renforcement d'une position dominante ou par création ou renforcement d'une puissance d'achat qui place les fournisseurs en situation de dépendance économique ». Les recommandations du Conseil de la Concurrence dans son avis de 1997, qui faisaient partie de la jurisprudence, sont donc maintenant intégrées à la loi, qui prend désormais en compte l'influence d'une concentration sur les relations verticales au même titre que son influence sur la concurrence horizontale.

Il est actuellement difficile de prévoir si cette loi parviendra à améliorer l'efficience de la régulation économique des relations commerciales. Les nouvelles règles ont été élaborées pour pallier le manque de précision des textes précédents, et en particulier de la loi Galland ${ }^{54}$. Cependant, rien dans cette loi ne vient corriger les inefficacités de la loi Raffarin évoquées précédemment. Elles permettront peut-être d'adapter les moyens réglementaires aux objectifs fixés par les pouvoirs publics. Mais une réflexion sur ces objectifs serait sans doute nécessaire. En effet, les différentes modifications réglementaires semblent refléter plus une volonté politique de satisfaire certains groupes de pression, qu'une véritable analyse économique de la situation ${ }^{55}$. Tout d'abord, il serait utile de préciser les objectifs fixés à la réglementation, qui semblent parfois contradictoires. Et avant tout, il convient de séparer les problèmes des relations de la grande distribution d'une part avec ses fournisseurs, d'autre part avec les autres formes de commerce concurrentes. Ensuite, il est nécessaire de vérifier le bienfondé économique de ces objectifs. Il faudrait en particulier que les pouvoirs publics approfondissent leur analyse économique de la situation, en prenant aussi en compte les

\footnotetext{
${ }^{53}$ Cette Commission n'a été effectivement mise en place qu'au premier semestre 2002.

${ }^{54}$ Les pouvoirs publics poursuivent actuellement leur réflexion afin d'amélioration la réglementation, notamment en ce qui concerne l'encadrement des marges arrières.

${ }^{55}$ Voir à ce sujet Rey et Tirole (2000).
} 
arguments qui plaident en faveur de la grande distribution, notamment en termes de coûts et d'efficacité. Enfin, le législateur peut également prendre en compte des arguments de politique industrielle: ainsi, un cadre réglementaire trop contraignant au niveau national sans harmonisation à l'échelle au moins européenne risque de créer des distorsions commerciales entre les différents acteurs, et notamment d'inciter les distributeurs français à privilégier les fournisseurs de pays concurrents. En particulier, les pouvoirs publics se penchent actuellement sur l'encadrement des marges arrières.

Enfin, l'émergence de nouvelles problématiques avec le développement du commerce électronique et l'apparition des places de marché « business to business » suscite un besoin nouveau d'adapter la réglementation. Ces places de marché, telles que GNX (Global Net Exchange) créé en février 2000, et WWRE (World Wide Retail Exchange), créé au printemps 2000, qui regroupent les plus grands distributeurs mondiaux, ont pour rôle de faciliter les transactions entre fournisseurs et distributeurs par la création d'un site proposant une standardisation des informations et des contrats d'échange. D'après certaines estimations, les transactions sur ces places de marchés devraient représenter d'ici 2004 30\% du commerce entre producteurs et distributeurs ${ }^{56}$. En améliorant la circulation de l'information et facilitant ainsi les comparaisons des offres des fournisseurs, ces plates formes renforceront probablement encore la puissance d'achat des distributeurs, en raison du déséquilibre entre le grand nombre de producteurs et le faible nombre de distributeurs adhérents. Il apparaît nécessaire d'étendre le dispositif réglementaire visant à encadrer les relations entre producteurs et fournisseurs aux transactions qui se déroulent sur ces places de marché. Toutefois, une telle réglementation doit se mettre en place à un niveau international, ce qui nécessite une coordination à long terme entre les Etats.

\section{Lexique}

Biens de consommation courante : «les biens offerts aux consommateurs peuvent être classés en deux grandes catégories : ceux qui, en raison de leur nature (eau, gaz, électricité) ou de leur "statut» (tabac, presse, médicaments, automobiles) font l'objet d'un système de distribution spécifique, et les autres qui sont désignés sous le terme de biens de consommation courante » (définition du Conseil de la Concurrence).

Business to business : Relations commerciales qui s'établissent sur Internet d'entreprise à entreprise.

Distribution exclusive : Contrat de distribution interdisant aux distributeurs de proposer des produits concurrents.

Distribution sélective : Mode de distribution dans lequel les produits du fabricant ne sont présents que chez certains détaillants choisis selon un cahier des charges fixé par le producteur, pour leurs compétences professionnelles et/ou leurs conditions d'installation.

Forclusion : Exclusion totale ou partielle du marché. La forclusion résulte d'une modification des conditions d'approvisionnement ou de vente, voire d'une rupture de la relation commerciale, et peut entraîner la cessation d'activité d'une firme.

\footnotetext{
${ }^{56}$ Source : LSA 15.03.2001.
} 
Linéaire : Longueur de rayons consacrée à l'exposition des produits dans un point de vente.

Marge arrière : Différence entre le prix unitaire facturé par le fournisseur et le prix réellement payé par le client, résultant des avantages accordés au client après la facturation.

Marge avant : Différence entre le prix le prix de détail et le prix facturé par le fournisseur. La marge totale du distributeur est la somme des marges avant et arrière.

Marque de distributeur (MDD) : produit qui porte la marque du distributeur et non celle du fabricant.

Maxidiscompte : Format de vente proposant aux consommateurs un assortiment restreint de produits à prix faibles, et offrant des services commerciaux minimaux.

Primes de référencement : Primes fixes versées par un producteur à un distributeur pour obtenir le référencement de ses produits.

Produits d'appel : Produits offerts à un prix faible et donnant lieu à une publicité visant à attirer les clients dans un point de vente.

Rabais : Réduction exceptionnelle sur le prix d'achat préalablement convenu accordé pour tenir compte d'un défaut de qualité ou de conformité des objets vendus.

Référencement / déréferencement: Le référencement est l'opération par laquelle un distributeur ou une centrale d'achat enregistre le(s) produit(s) d'un fournisseur dans son catalogue. Cette action ne constitue pas un engagement d'achat mais une condition préalable nécessaire pour qu'une relation commerciale effective soit établie. Le déréférencement est la résiliation du contrat de référencement.

Remise : Réduction de prix.

Ristourne : Réduction de prix différée accordée après l'établissement de la facture.

Têtes de gondole : Etagères situées à l'extrémité des rayons, sur l'allée centrale par exemple, et qui permettent de mettre en évidence des articles lors de campagnes promotionnelles.

Vente liée : Vente d'un produit soumise à l'achat concomitant d'un autre produit.

Zone de chalandise : Zone géographique dans laquelle résident les clients d'un magasin.

\section{Bibliographie}

AFEC (1999) «Ententes et concentrations : les différences de traitement juridique sont-elles justifiées? », rapport du groupe de travail de l'AFEC, 8 avril 1999.

Allain, M.-L. (2000) «Structures de marché et réglementation : les fondements des rapports de force entre producteurs et distributeurs ", Thèse de doctorat EHESS.

Allain, M.-L. et L. Flochel (2001) « Contrainte de capacité et développement des marques de distributeurs », Revue Economique Vol. 52 n³, 643-653.

Bergès Sennou, F. (2001) «L'analyse économique des pratiques commerciales dans l'industrie agroalimentaire », Thèse de doctorat Université de Toulouse.

Bliss, C. (1988), "A Theory of Retail Pricing", The Journal of Industrial Economics, 36,4.

Bontems, P., S. Monnier et V. Réquillart (1998) «Strategic Effects of Private Labels », European Review of Agricultural Economics, 6, 147-165.

Boutard-Labarde, M.-C. et G. Canivet (1994) « Droit français de la concurrence », Librairie Générale de Droit et de Jurisprudence, Paris. 
Caprice, S. (2000) «Contributions à l'analyse de la puissance d'achat dans les relations verticales : interactions stratégiques et marques de distributeurs » Thèse de doctorat Université de Paris I.

Caprice, S. et J. Philippe (1997) «Les relations verticales", in "Réglementation et Concurrence », A. Perrot éd., Economica.

Chambolle, C. (2000) " Analyse théorique du rapport de force dans les relations verticales et applications au secteur agroalimentaire », Thèse de doctorat Université de Paris I.

Chambolle, C. (2001) «Faut-il interdire la revente à perte?», à paraître dans la Revue Française d'Economie.

Charléty, P. et S. Souam (2000) «Les concentrations dans l'industrie française », in «La dynamique concurrentielle en France », LEI et EUREQua, Rapport pour le Commissariat au Plan.

Dobson, P. Waterson, M. Clarke, R. et S. Davies (1999), "Buyer Power and its Impact on Competition in the Food Retail Distribution Sector of the European Union", Publication des Communautés Européennes

Farrell, J. et C. Shapiro (1990) «Horizontal Mergers : an Equilibrium Analysis », American Economic Review, 80 (1) 107-126.

Galène, R. (1994) «Le droit de la concurrence appliqué aux pratiques anticoncurrentielles », Litec.

Kamien, M. et I. Zang (1993) « Monopolization by sequential acquisition », Journal of Law, Economics and Organisation 9 (2) p205-229.

Klein, B., R. Crawford et A. Alkian (1978) « Vertical Integration, Appropriable Rents and the Competitive Contracting Process », Journal of Law and Economics, 21, 297-326.

Lang, J. T. (2000) «International Joint Venture under Community Law », Fordham Corporate Law Institute, Twenty-sixth annual conference on International Antitrust Law and Policy.

Milgrom, P. et J. Roberts (1982), « Limit Pricing and Entry Under Incomplete Information », Econometrica, 50, p. 443-460.

Mills, D. (1995) "Why retailers sell private labels", Journal of Economics and Management Strategy, 4 (3), 509-528.

Ordover, J., G. Saloner et S. C. Salop (1990) « Equilibrium Vertical Foreclosure », American Economic Review, 80 (1), 127-142.

Philippe, J. (1997) "Relations producteur-distributeur : les enseignements du secteur de l'habillement", Economie et Statistique, 303, 101-112.

Philippe (1997) «L'accès au linéaire : une ressource essentielle ? » Revue de la Concurrence et de la Consommation, 100, 12-15.

Philippe, J. (1998) «La mesure du marché pertinent », Revue Française d'Economie, 13 (4), 125-159.

Rey, P. (1997) «Impact des accords verticaux entre producteurs et distributeurs », Revue Française d'Economie, 12 (2), 3-55.

Rey, P. et J. Tirole (1997) «Analyse économique de la notion de prix de prédation » Revue Française d'Economie 12, p3-32. 
Rey, P. et J. Tirole (2000) « Quelles régulations pour le commerce ? », rapport pour le Conseil d'Analyse Economique n²9, La Documentation Française.

Tirole, J. (1988) « The Theory of Industrial Organization », MIT Press.

Werden, G.J. et L.M Froeb, (1994), "The Effects of Mergers in Differentiated Products Industries: Logit Demand and Merger Policy", The Journal of Law, Economics, \& Organization, 10, 2, pp. 407-437. 